\title{
Application of Singular Spectrum Analysis in Deep-Ocean Tide Reconstruction and Prediction
}

\author{
Peeravit KOAD ${ }^{1,2, *}$ and Krisanadej JAROENSUTASINEE ${ }^{1,2}$ \\ ${ }^{1}$ School of Science, Walailak University, Nakhon Si Thammarat 80160, Thailand \\ ${ }^{2}$ Center of Excellence for Ecoinformatics, Walailak University, Nakhon Si Thammarat 80160, Thailand
}

('Corresponding author's e-mail: harrykoad@gmail.com)

Received: 2 July 2019, Revised: 2 October 2019, Accepted: 2 November 2019

\begin{abstract}
This study utilized the Singular Spectrum Analysis (SSA) approach to perform time series orthogonalization and demonstrated its use by analyzing vertical tidal acceleration and sea level time series from different deep-ocean locations. This method quantifies astronomical variations by using decomposed vertical tidal acceleration to reconstruct and predict deep-ocean tide. The results show that each decomposed vertical tidal acceleration can be associated with the decomposed sea level having at least 5 astronomical variations. Their associated energies can also be used to diagnose the change of the oceanic tide response to tidal acceleration. Performance evaluation also shows that this method can give comparable reconstruction accuracy and slightly better prediction accuracy compared to the harmonic analysis-based method. It is indicated that the proposed method is accurate enough to be applied in a tsunami detection algorithm. The results also indicate that the proposed method is stable enough to provide unpropagated prediction residuals.
\end{abstract}

Keywords: Singular spectrum analysis, Empirical orthogonal function, Principal component analysis, Tidal acceleration, Deep-ocean tide

\section{Introduction}

The oceanic tide, as the changing of sea level during the day, is one of the oldest geophysical time series ever recorded by humans. The fluctuation of the oceanic tide was found to correlate with apparent positions of the Moon and the Sun in the sky in ancient times. Ever since the scientific community has paid attention to its impact. For example, the oceanic tide was found to correlate well with smallmagnitude earthquakes in some areas, which suggests that tidal triggering may be their cause $[1,2]$. The oceanic tide plays a vital role in the maritime environment, such as by modulating the motion of ice sheets near marine margins [3,4], manipulating the oceanic circulation [5,6], and influencing the effect of sea level rise $[7,8]$. Oceanic tide was also used to study the Earth's paleoclimate and paleoceanography and the evolution of the lunar orbit $[9,10]$.

An approach that can be used to predict the oceanic tide empirically is based on the concept of the harmonic method and the Principal Tidal Constituents (PTCs). The harmonic method basically uses a set of PTCs with predefined frequencies to perform the least-squares harmonic analysis, then apply some corrections for nodal modulation (variations in lunar nodal progression) or satellite modulation (which also includes variations in other lunar and solar arguments) to account for long-term prediction [11,12].

These nodal (or satellite) corrections, which are used to adjust amplitudes and phases of the fitted PTCs to account for their nearby unresolved frequencies, can reduce the magnitude of this problem and give accurate prediction results of up to several years in advance. However, this approach still cannot give correct frequencies since orbital arguments of the Moon and the Earth change slowly with time over several decades [11]. 
http://wjst.wu.ac.th

The traditional harmonic method, which includes nodal corrections, being used extensively in oceanic tide analysis emerges directly from the tidal potential theory first formalized by Doodson in 1921 and simplified by Godin in 1972 [11,13]. This method assumes that a sea level time series can be expressed as a summation of $n$ sinusoidal functions as;

$$
Z\left(t_{i}\right)=Z_{0}+\sum_{j=1}^{n} a_{j}\left(t_{0}\right) A_{j} \cos \left(2 \pi f_{j}\left(t_{i}-t_{0}\right)+v_{j}\left(t_{0}\right)+u_{j}\left(t_{0}\right)-\phi_{j}\right)+R\left(t_{i}\right)
$$

where $Z\left(t_{i}\right)$ is the observed sea level at time $t_{i}, Z_{0}$ is the average sea level, $a_{j}\left(t_{0}\right)$ and $u_{j}\left(t_{0}\right)$ are the nodal corrections to amplitude and phase at a reference time $t_{0}$ respectively, $v_{j}\left(t_{0}\right)$ is the astronomical offset, and $R\left(t_{i}\right)$ is the residuals.

A least-squares approach is applied to estimate the values of $Z_{0}, A_{j}$, and $\phi_{j}$, where $t_{i}$ are usually assumed to be regularly sampled. Notice that the values of $a_{j}\left(t_{0}\right), u_{j}\left(t_{0}\right)$, and $v_{j}\left(t_{0}\right)$ are also assumed to be constants, which is unnatural since the astronomical arguments always change with time.

A more recently developed method called the Versatile Harmonic Tidal Analysis (VHTA) has eliminated these limitations (regular sampling and constant nodal corrections) by relaxing the constancy of phases to be dependent with time, in which the frequency and phase of each PTC is combined as a temporal function $V_{j}\left(t_{i}\right)$ to be computed from the observed sea level data [11] as;

$$
Z\left(t_{i}\right)=Z_{0}+\sum_{j=1}^{n} a_{j}\left(t_{i}\right) A_{j} \cos \left(V_{j}\left(t_{i}\right)+u_{j}\left(t_{i}\right)-\phi_{j}\right)+R\left(t_{i}\right)
$$

while the values of $a_{j}\left(t_{i}\right)$ and $u_{j}\left(t_{i}\right)$ are not constants, and the values of $Z_{0}, A_{j}$, and $\phi_{j}$ can be estimated using the Singular Value Decomposition (SVD).

Although the determination of $V_{j}\left(t_{i}\right)$ eliminates the presence of the problem of significant gaps, another problem with the number of PTCs to be used in the harmonic method still remains. Using too many PTCs causes the estimated amplitudes of most of them to be lower than a certain confidence level of the red noise spectrum fitted to the observed sea level time series. In practice, the number of sinusoidal functions that can be significantly estimated using the harmonic analysis, with no a priori PTCs being used, is typically less than 30. For example, only 17 significant PTCs were detected in a sea level time series at Workington, England [14,15]. This is evidence that using more numbers of PTCs in predicting oceanic tide always gives more accurate results where some of them are not significant.

In 1966, Munk and Cartwright proposed a new method of predicting oceanic tide using the precomputed TGP time series called the response method [16-18]. This method computes the gravitational and radiation potentials from the position of the Moon and the Sun then decomposes them using the spherical harmonic expansion. Spherical harmonic modes $Y_{n}^{m}$ are spatially (but not temporally) orthogonal, and the value of each mode at a given position and time is a complex number consisting of both the real and imaginary parts. The Linear Least-Squares (LLS) is used to estimate coefficients for both the real and imaginary parts of the selected spherical harmonic modes, including their past and present values, such that the residuals between the actual sea level time series and their linear combination are minimized.

The response method proposed that the local gravitational potential $P(\theta, \lambda, t)$ at co-latitude $\theta$, longitude $\lambda$, and time $t$ can be expressed in the spherical harmonic formalism as;

$$
P(\theta, \lambda, t)=g \sum_{n=0}^{\infty} \sum_{m=-n}^{n}\left(U_{n}^{m}(\theta, \lambda) a_{n}^{m}(t)+V_{n}^{m}(\theta, \lambda) b_{n}^{m}(t)\right),
$$


http://wjst.wu.ac.th

where $g$ is the acceleration due to gravity, assumed to be a constant, $Y_{n}^{m}(\theta, \lambda)=U_{n}^{m}(\theta, \lambda)+i V_{n}^{m}(\theta, \lambda)$ is a constant complex spherical harmonic expansion at a location on Earth (where $i$ is an imaginary number), and $c_{n}^{m}(t)=a_{n}^{m}(t)+i b_{n}^{m}(t)$ is a spherical harmonic expansion of the combined gravitational potential from the Moon and the Sun at time $t$.

The term $c_{n}^{m}(t)$ associated with the position of the Moon and the Sun at time $t$ can be separated from $Y_{n}^{m}(\theta, \lambda)$ to create the global gravitational tide-generating function $P(t)$. This function can be defined as;

$$
P(t)=g \sum_{n=0}^{\infty} \sum_{m=-n}^{n}\left(a_{n}^{m}(t)+b_{n}^{m}(t)\right)
$$

In the case of using only $P(t) / g$ as predictors, the response method assumes that a sea level time series can be written as;

$$
Z(t)=Z_{0}+\sum_{n=2}^{3} \sum_{m=0}^{n} \sum_{s=-3}^{3}\left(u_{n}^{m}(s) a_{n}^{m}(t-s \Delta \tau)+v_{n}^{m}(s) b_{n}^{m}(t-s \Delta \tau)\right)+R(t)
$$

where $w_{n}^{m}(s)=u_{n}^{m}(s)+i v_{n}^{m}(s)$ is the coefficient or prediction weight (called impulse response) to be estimated using a least-squares method and $s \Delta \tau$ is the time lag. Notice that the number $n$ runs from 2 to 3 , which in practice means the upper limit can be greater than 3 and, in the case of radiation potential, the lower limit can start from 1 . The number $m$ runs from 0 to $n$ because of the identity $Y_{n}^{-m}=(-1)^{m} Y_{n}^{m *}$; therefore, the prediction function can be simplified by omitting negative values of $m$, as in equation (5). The values of $U_{n}^{m}(\theta, \lambda)$ and $V_{n}^{m}(\theta, \lambda)$, which are constants and coefficients for the negative $m$, can also be lumped into the values of $u_{n}^{m}(t)$ and $v_{n}^{m}(t)$.

The numbers $n$ and $m$ can also be used to describe the nature of each spherical harmonic mode. The values of $m=0,1$, and 2 represent the long-period, diurnal, and semidiurnal constituents, respectively, In the case of the gravitational potential, solar variations of $S_{a}$ and $S_{1}$ were found to dominate for $n=0$ and 1. For $n=2$, variations of the Moon and the Sun were found to have an amplitude ratio of 5:2 and contain major variations of $M_{f m}, M_{f}, M_{m}, S_{s a}$, and $M_{n}$ (for $m=0$ ), $K_{1}, P_{1}, O_{1}$, and $Q_{1}$ (for $m=1$ ), and $K_{2}, S_{2}, M_{2}$, and $N_{2}$ (for $m=2$ ). The rest of the spherical harmonic modes, having $n \geq 3$, were found to be dominated by lunar variations.

However, Groves and Reynolds pointed out in their work that the time functions used in equation (5) are not orthogonal, a method having both the smoothness of admittances (the Fourier transform of impulse responses) and orthogonality that would be superior [19]. Some modifications have been applied to impulse responses such that all of the orthogonal tidal inputs (called linear orthotides in their work) are orthogonalized.

The response method can be used to analyze both long-term and short-term tidal records and was found to provide better reconstruction and prediction than the conventional harmonic method in some cases. However, this method requires computing perturbations to the gravitational potential caused by the Sun and the Moon, while the conventional harmonic method uses predetermined PTCs and nodal corrections (or a combination of them in the form of polynomials derived from astronomical ephemerides for the VHTA), which are often more convenient to use to perform tidal reconstruction and prediction 
http://wjst.wu.ac.th

compared to the response method. Moreover, the fact that the harmonic method has been commonly and widely used for a long time makes it more popular for oceanic tide analysis than the response method.

The Principal Component Analysis (PCA) has received much attention for several decades in data analysis using a covariance matrix [20]. PCA transforms the original data into a set of the Principal Components (PCs), which requires only a few examples to optimally reconstruct original data. Moreover, each eigenvalue that is associated with the explained variance can be used to quantitatively indicate its contribution to overall variations in the original data.

An extension of PCA which is widely used in time series analysis is called the Singular Spectrum Analysis (SSA) [21]. The SSA generally obtains spectral information through the covariance matrix and eigensystem (eigenvectors and their associated eigenvalues), computed from a time series. The mathematical formulation of time series orthogonalization based on the SSA approach, which serves as the basis of reconstruction and prediction methods, will be given later.

Due to the advantage of the response method using astronomical ephemerides which are computable as input data, and the concept of data orthogonalization, inspired by the SSA approach, this study utilizes the concept of the response method and combines it with a slight modification of the SSA approach. This study aims at (1) utilizing the SSA approach as the time series orthogonalization method to analyze tidal acceleration and sea level time series, in order to quantify astronomical variations, and (2) using orthogonalized tidal acceleration to predict deep-ocean tide, as well as to evaluate its performance.

\section{Materials and methods}

\section{Data acquisition}

\section{a. Sea level data}

Astronomical variations drive the oceanic tide observed in sea level time series [17]. In this study, the sea level time series is measured as the height of the sea surface with respect to a referenced depth relative to the seafloor. To test whether the proposed technique is suitable for applications that require accurate oceanic tide reconstruction and prediction, such as tsunami detection, sea level data from the National Data Buoy Center (NDBC) were used. These data were collected by bottom pressure recorders deployed together with moored buoys operated by the Deep-ocean Assessment and Reporting of Tsunamis (DART) Project. Deep-ocean data collected by DART stations were downloaded, and only data from 57 stations with a long enough time span (usually from 2005 to 2017) of the available records were selected (Figure 1).

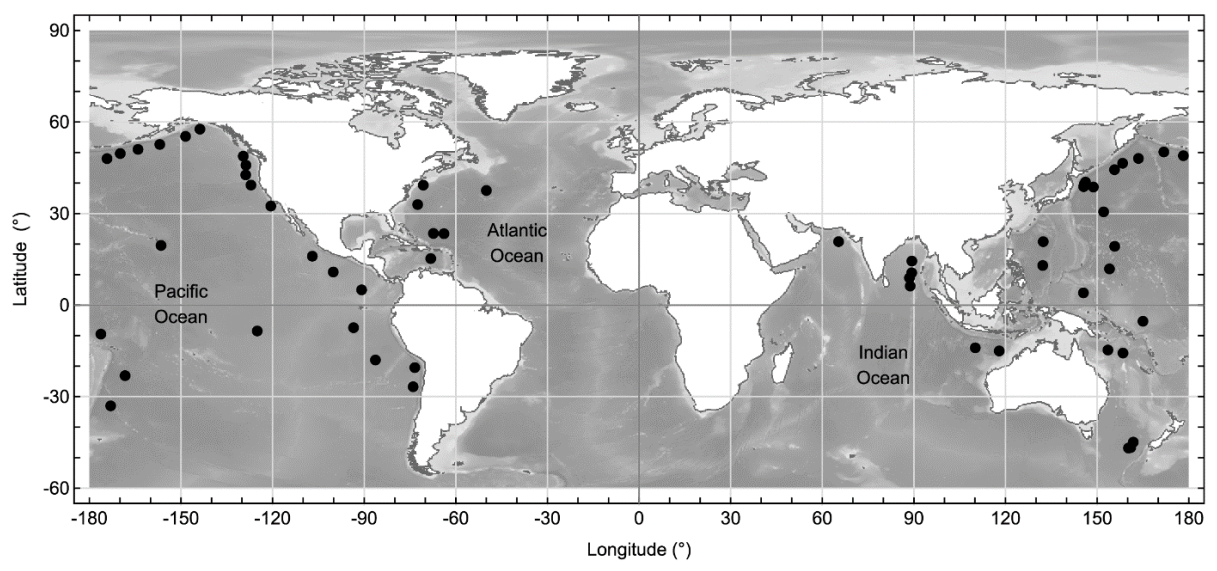

Figure 1 Locations of the selected 57 bottom pressure recorder stations using the DART system from the National Oceanic and Atmospheric Administration (NOAA) (dots). 


\section{b. Vertical tidal acceleration data}

TGP derived from the Newton-Kepler dynamics can be used as the input time series for the oceanic tide, as described in [18,22]. In fact, this potential is a scalar function, and using the tidal acceleration (which is a vector function) seems to be more reasonable than using the tidal potential since the true cause of the motion is the acceleration.

The primitive astronomical data, which consisted of apparent geocentric positions of the Moon, the Sun, and planets, were computed at the 57 DART stations. In this study, orbital parameters of celestial objects from NASA JPL's DE405 solar system ephemeris were used [23]. This ephemeris was directly derived from the results of numerical integration of celestial dynamics, which can be considered to be more accurate than a set of orbital arguments computed using a set of polynomials used by the response method in $[18,22]$.

The tidal acceleration vector can be computed using Newton's law of universal gravitation as the difference in acceleration due to gravity at the surface and center of the Earth. The acceleration due to gravity $\vec{a}_{O X}$ from a celestial object $X$ of mass $M_{X}$ which is exerted on an observer on the surface of the Earth at $O$ along a position vector $\vec{r}_{O X}$ from $O$ to $X$ can be computed from;

$$
\vec{a}_{O X}=\frac{G M_{X}}{r_{O X}^{2}} \hat{r}_{O X}=\frac{G M_{X}}{r_{O X}^{3}} \vec{r}_{O X},
$$

and the acceleration $\vec{a}_{C X}$ from the center of the Earth at $C$ to $X$ along $\vec{r}_{C X}$ can be computed from;

$$
\vec{a}_{C X}=\frac{G M_{X}}{r_{C X}^{2}} \hat{r}_{C X}=\frac{G M_{X}}{r_{C X}^{3}} \vec{r}_{C X}
$$

Individual tidal acceleration from $X$ at $O$ is therefore equal to $\vec{a}_{O X}-\vec{a}_{C X}$, and in the case of $N$ celestial objects $(i=1$ to $N$ ) the general tidal acceleration $\vec{T}$ at $O$ can be computed from;

$$
\vec{T}=G \sum_{i=1}^{N} M_{X_{i}}\left(\frac{1}{r_{O X_{i}}^{3}} \vec{r}_{O X_{i}}-\frac{1}{r_{C X_{i}}^{3}} \vec{r}_{C X_{i}}\right) .
$$

This tidal acceleration was converted into the topocentric horizontal coordinate system. Tidal variations which are not originated from tidal acceleration from the Moon and the Sun, such as internal tides found over ocean ridges and the continental shelf, or gravity waves caused by differences in densities of seawater, cannot be fully reconstructed or predicted, since these variations are location specific and not directly caused by astronomical phenomena [12,13,18]. As a consequence, tidal variations caused by these local conditions will be contained in reconstruction and prediction residuals. Therefore, this study focuses on deep-ocean tide analysis, in which the effects of bathymetry can be ignored in practice; only its vertical component (zenith-nadir direction) was used henceforth as the Vertical Tidal Acceleration (VTA).

The VTA time series at each DART station was computed on the surface of the WGS84 reference ellipsoid, which is currently used to represent the flattening of the Earth. This ellipsoid can be considered as the "theoretical mean sea level", which is subjected to the rotation of the Earth without the influence of tidal forces from the Moon and the Sun. Although the actual mean sea level at a station can differ from the reference ellipsoid, this difference is generally small compared to variation in the computed VTA and can be neglected in practice. 


\section{Data analysis}

\section{a. Central moving average}

In order to account for any irregular trend which is of non-astronomical origin, several detrend methods can be used to obtain the low-pass signal. However, to maintain the flexibility of the time series orthogonalization method proposed in this study, the central moving average, which seems to be the simplest detrend method, was used.

The central moving average was only performed on sea level time series obtained from the 57 DART stations, not on their VTA time series. A major problem of using these sea level data is significant drifting of values measured by instruments, which may be originated from the loose deployment of instruments, or redeployment at a new location after station maintenance. Therefore, in this study, a onemonth central moving average was performed on all sea level time series to detrend the long-term trend. Any variations with periods of more than 1 month were also removed as a consequence.

Each detrended sea level time series was split into training and testing data. All records, except for the last year, were used in time series orthogonalization, in order to be analyzed for astronomical variations and to be used in deep-ocean tide reconstruction. The last year in each detrended time series was reserved as the testing data to be used in tidal prediction.

Sea level data from a DART station with WMO number 23219 (hereafter DART 23219), along with its VTA data, were used to demonstrate the methodology. This DART station was deployed at a distance of $350 \mathrm{~km}$ to the west of the Andaman and Nicobar Islands in the central region of the Bay of Bengal in the Indian Ocean (latitude $10^{\circ} 35^{\prime} \mathrm{N}$, longitude $89^{\circ} 17^{\prime} \mathrm{E}$ ). The sea level time series of this station was detrended (Figure 2 (a)). The VTA time series was also computed at the same location (Figure 2 (b)).

(a)

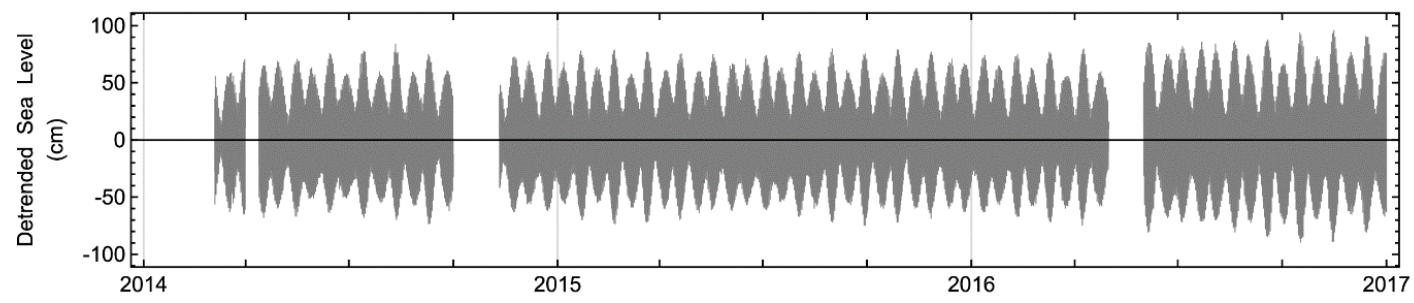

(b)

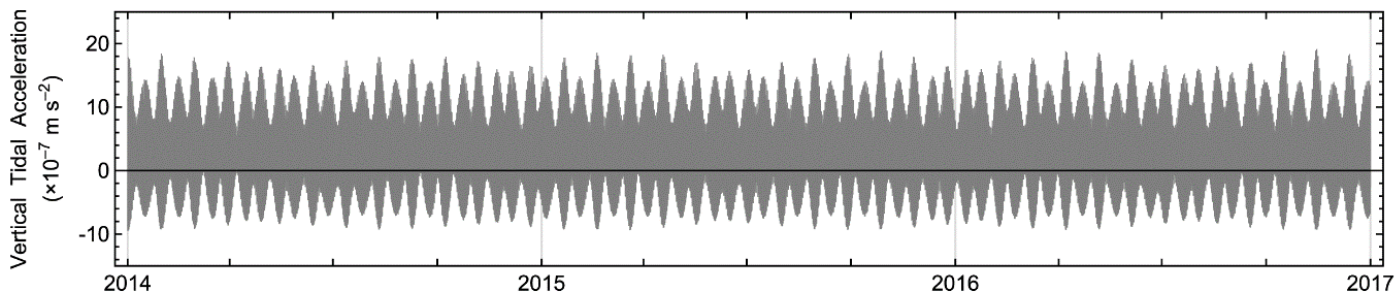

Figure 2 Time series plots of the training sea level (a) and the VTA (b) of DART 23219 deployed at the central region of the Bay of Bengal in the Indian Ocean. Sea level data contained 3 large gaps due to sensor malfunctions and was redeployed in 2016 following the last gap. The randomly selected sea level fragments which contained missing records from these gaps were excluded from the analysis. The recorded sea level following the sensor redeployment since June 2016 had a slightly higher variance, which caused the prediction residuals at this station to be increased. 


\section{b. Time series orthogonalization}

Time series orthogonalization based on the SSA approach was performed on the time series of the computed VTA, along with the training sea levels at the 57 DART stations. Each time series was randomly selected for $n$ fragments of equal time span (having $p$ records in each fragment). These fragments could be overlapped in parts, and fragments that contained missing data were not selected.

This set of fragments is an $n \times p$ matrix $\mathbf{X}$ which can be decomposed into 3 composite matrices, namely $n \times n$ orthogonal matrix $\mathbf{U}, n \times p$ diagonal matrix $\mathbf{W}$, and $p \times p$ orthogonal matrix $\mathbf{V}^{\mathrm{T}}$, using SVD $[20,24,25]$ by;

$$
\mathbf{X}=\mathbf{U W} \mathbf{V}^{\mathrm{T}} .
$$

The leading diagonal elements of $\mathbf{W}$ contains a rank-ordered set of singular values (from larger to smaller ones) or the square root of eigenvalues of the covariance matrix of $\mathbf{X}$. $\mathbf{U}$ and $\mathbf{V}$ contain the left and right singular vectors, which by definition are eigenvectors of $\mathbf{X} \mathbf{X}^{\mathrm{T}}$ and $\mathbf{X}^{\mathrm{T}} \mathbf{X}$, respectively.

In meteorological literature, $\mathbf{U}$ and $\mathbf{V}$ are also called spatial and temporal EOFs, respectively, in which the use of the first $k$ EOFs $(1 \leq k \leq \min (n, p))$ leads to an optimal approximation of $\mathbf{X}[20,24,25]$. Projection of each row in $\mathbf{X}$ onto an orthonormal basis, defined by EOFs (columns in V), is equivalent to finding PCs $\mathbf{X}_{P C}$ as;

$$
\mathbf{X}_{P C}=\mathbf{X V} \text {. }
$$

Each PC and its associated EOF has its own explained variation, hereafter called the "energy" (the word "energy" is a conventional technical term used extensively in EOF analysis and is not to be confused with the "spectral energy density" of each frequency in signal processing, which is the absolute square of the Fourier transform of a time series).

A measure of how well these first $k$ EOFs can represent the original matrix can be computed as cumulative eigenvalues of each $i^{\text {th }}$ singular value $\sigma_{i}$ (or the $i^{\text {th }}$ diagonal element in $\mathbf{W}$ ), hereafter called the "total energy" $\Lambda_{k}$, by;

$$
\Lambda_{k}=\sum_{i=1}^{k} \sigma_{i}^{2} .
$$

$\sigma_{i}$ relates to the $i^{\text {th }}$ eigenvalue of $\mathbf{X}^{\mathrm{T}} \mathbf{X}\left(\right.$ or $\left.\lambda_{i}\right)$ and the covariance matrix $\mathbf{C}_{\mathbf{X}}$ as;

$$
\mathbf{C}_{\mathbf{X}} \mathbf{v}_{i}=\frac{\mathbf{X}^{\mathrm{T}} \mathbf{X}}{n-1} \mathbf{v}_{i}=\frac{\lambda_{i}}{n-1} \mathbf{v}_{i}=\frac{\sigma_{i}^{2}}{n-1} \mathbf{v}_{i},
$$

where $\mathbf{v}_{i}$ is the $i^{\text {th }}$ temporal eigenvector or the $i^{\text {th }}$ column of $\mathbf{V}$.

$\Lambda_{k}$ is a measure of variation explained by the first $k$ EOFs compared to the variation of the original time series. It is usually expressed as a ratio $\Lambda_{k} / \Lambda_{p}$ and its percentage and is often used as a criterion for deciding the number of EOFs to retain for the low-rank approximation of $\mathbf{X}$.

The low-rank approximated matrix $\mathbf{X}^{\prime}$, which contains the smoothed fragments from the original fragment matrix $\mathbf{X}$, can be obtained directly using a transformation matrix $\mathbf{B}_{\mathbf{X}, \mathbf{X}^{\prime}}$ which uses the modified $p \times p$ identity matrix $\mathbf{I}^{\prime}$ (by setting diagonal elements from row $k+1$ to $p$ to zeros) by;

$$
\mathbf{X}^{\prime}=\mathbf{X V I} \mathbf{I}^{\prime} \mathbf{V}^{\mathrm{T}}=\mathbf{X B}_{\mathbf{X}, \mathbf{X}^{\prime}} .
$$


A criterion for selecting elements in $\mathbf{I}^{\prime}$ to be replaced with zeros depends on total energy, as by equation (11); then, $\mathbf{B}_{\mathbf{X}, \mathbf{X}^{\prime}}$ is constructed with the use of $\mathbf{V}$. The signal-to-noise separation can also be visualized in a scree plot in which the first $k$ EOFs before the discontinuity of their energies are kept [20,24,25] (Figure 3 (a) to (d)).

Each element in the smoothed fragment in $\mathbf{X}^{\prime}$ is equivalent to a weighted moving summation value from every element in the same fragment in $\mathbf{X}$. This means every column in $\mathbf{B}_{\mathbf{X}, \mathbf{X}^{\prime}}$ serves as a different version of weights according to positions of elements in each fragment. These weights as row vectors can be treated as correlation kernels to be correlated with a time series in order to perform time series approximation. In this study, the central column in $\mathbf{B}_{\mathbf{X}, \mathbf{X}^{\prime}}$ was used as a correlation kernel.

$\mathbf{B}_{\mathbf{X}, \mathbf{X}^{\prime}}$ is also a separable matrix which consists of $k$ square matrices $\mathbf{F}_{i}$, each with the dimension of $p \times p$. Each $\mathbf{F}_{i}$ is a self-outer-product (or an unscaled autocorrelation matrix) of the $i^{\text {th }}$ temporal eigenvector $\mathbf{v}_{i}$ as;

$$
\mathbf{B}_{\mathbf{X}, \mathbf{X}^{\prime}}=\mathbf{V I}^{\prime} \mathbf{V}^{\mathrm{T}}=\sum_{i=1}^{k} \mathbf{v}_{i} \otimes \mathbf{v}_{i}=\sum_{i=1}^{k} \mathbf{F}_{i} .
$$

Similar to $\mathbf{B}_{\mathbf{X}, \mathbf{X}}$, every $\mathbf{F}_{i}$ serves as a different version of the transformation matrix in which their central columns can also be used as correlation kernels to be correlated (the weighted central moving average) with a time series in order to perform time series orthogonalization. An individual decomposed time series will hereafter be called an "eigenmode". All eigenmodes are instantaneously orthogonal, and summation of the first $k$ eigenmodes is the approximated time series (or is nearly equal to the original time series).

(a)

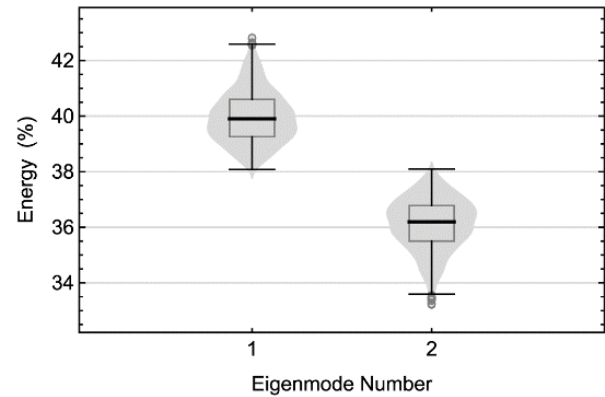

(c)

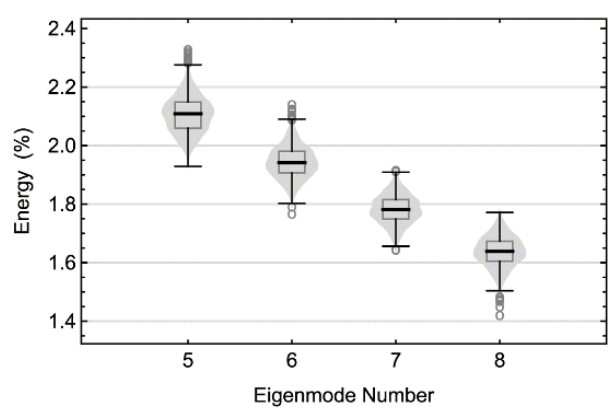

(b)

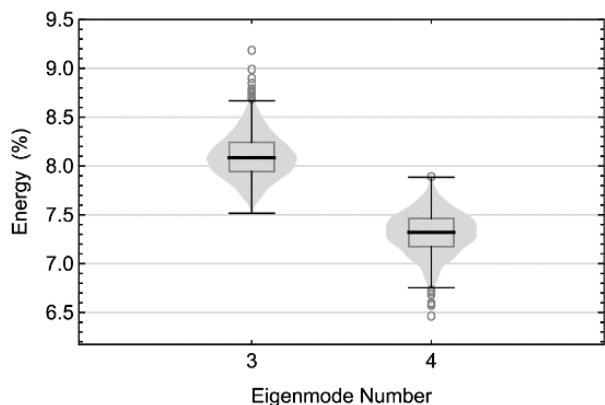

(d)

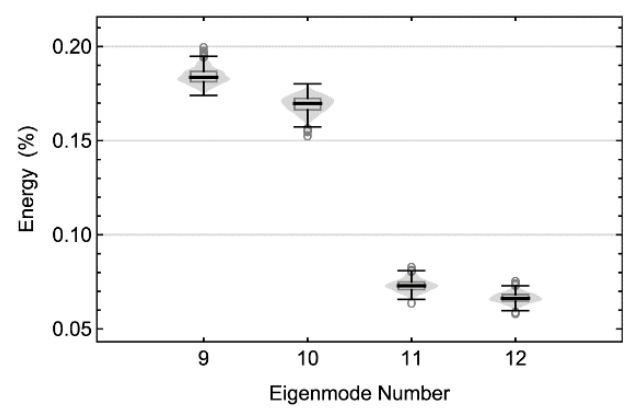

Figure 3 Scree plots showing energies of eigenmodes 1 and 2 (a), 3 and 4 (b), 5 to 8 (c), and 9 to 12 (d), estimated from 1,000 orthogonalizations (using different sets of the randomly selected fragment) of the training sea level time series of DART 23219. Eigenmodes can be grouped in pairs having similar values of energies, such as eigenmodes 1 and 2, which have energies of about $38 \%$, or eigenmodes 3 and 4 , with energies of about $8 \%$. The overlapping of eigenmodes can also be seen in some plots, such as that of eigenmodes 5 to 8 . 
http://wjst.wu.ac.th

The timespan of each fragment $p$ determines the longest variation which can be captured by the SSA [21,24]. The increase in $n$ or $p$ results in the convergence of eigenvalues and eigenvectors, making them more stable. In this study, the one-month central moving average was applied to sea level time series, which also removed astronomical cycles with periods of longer than a month. Therefore, the time span of fragments that were randomly selected during a time series orthogonalization process was set to 30 days to adequately cover monthly variations contained in both the VTA and the detrended sea level time series. After orthogonalization, the Discrete Fourier Transform (DFT), without performing the nodal correction (or more generally, the satellite modulation), was used as the harmonic analysis method to analyze the actual variations (without using knowledge of the predetermined astronomical variations) contained in these eigenmodes to study their patterns.

\section{c. Time series reconstruction and prediction}

The time series of the detrended sea level $C(t)$ at time $t$, which consists of $p_{C}$ sea level eigenmodes $C_{i}(t)$, can be approximated using the first $k_{C}$ eigenmodes; then, the summation of the rest of the eigenmodes is the residuals represented by $R_{C}(t)$. These can be written as;

$$
C(t)=\sum_{i=1}^{p_{C}} C_{i}(t)=\sum_{i=1}^{k_{C}} C_{i}(t)+R_{C}(t)
$$

The VTA time series $A(t)$, which consists of $k_{A}$ astronomical eigenmodes $A_{j}(t)$ and the residuals $R_{A}(t)$, can also be written in a similar fashion as;

$$
A(t)=\sum_{j=1}^{p_{A}} A_{j}(t)=\sum_{j=1}^{k_{A}} A_{j}(t)+R_{A}(t) .
$$

This study hypothesizes that, if the variation of a sea level time series $C(t)$ is caused by astronomical variations contained in the VTA time series, then one can use variations of some VTA eigenmodes $A_{j}$ to generate an oceanic tide $Z(t)$ such that $C(t)-Z(t)$ is minimized in the least-squares sense. The time series of oceanic tide $Z(t)$ can be estimated using the linearization in which a value of $A_{j}$ at time $t$ and a few past values at time $t-\Delta \tau, t-2 \Delta \tau, t-3 \Delta \tau, \ldots$, and $t-n_{s} \Delta \tau$, as well as their multipliers $m_{j, s}$, are incorporated together as;

$$
Z(t)=\sum_{j=1}^{k_{A}} \sum_{s=0}^{n_{s}} m_{j, s} A_{j}(t-s \Delta \tau) .
$$

In this study, the maximum time lag of 6 days, as found by Munk and Cartwright to be the most appropriate value (i.e., $\Delta \tau=1$ day and $s=0$ to 6), was used in this study [18]. The values of $m_{j, s}$ were estimated using LLS between the first $k_{A}$ VTA eigenmodes and the training sea level time series at the 57 DART stations.

This equation can be considered to be the linearized version of the linear combination of all possible solutions to a system of differential equations governing the oceanic tide, which already includes information of their derivatives through the incorporation of a number of their past values. Although this equation is not the actual complete solution, it is at least the first guess solution obtained directly from the observed time series. 
http://wjst.wu.ac.th

\section{d. Differences between methods}

The VHTA, as a representative of the harmonic method used to analyze oceanic tide time series in this study, computes PTCs and their satellite modulations from the predetermined Doodson numbers and the computed astronomical arguments of the Moon and the Sun (without computing their positions or tidal accelerations) at a specified time [11], while the proposed method in this study first computed astronomical data, then decomposed it into a set of the orthogonalized time series. Therefore, this study is more flexible, in the sense that the astronomical ephemerides computation procedure can be replaced by other, more accurate, versions, while astronomical variations used in the VHTA and other harmonic methods are fixed to Doodson numbers that cannot account for complex orbital perturbations.

The main difference between the method proposed in this study and the response method is that the latter uses LLS to directly fit spherical harmonic modes of gravitational and radiation potentials to only the original sea level time series [18], while this study used VTA eigenmodes instead of spherical harmonic modes. Moreover, the time series of some spherical harmonic modes were not found to be temporally orthogonal with each other, especially for time series of $a_{0}^{0}$ and $a_{1}^{0}$, while VTA eigenmodes are orthogonal.

Unlike the proposed method in this study, the fitting of spherical harmonic modes to the original sea level time series, as done by the response method, cannot estimate the energy of each mode, as can be done with the computation of eigenmodes. This problem is caused by the definition of eigenvalues in the spherical harmonic expansion, which is different from that of the SSA. In the case of the SSA, eigenvalues reflect the magnitude of the variance of each EOF in descending order [20,21,24,25], while eigenvalues in spherical harmonic expansion represent another meaning.

The workflows of the VHTA (the harmonic method) described in [11], the response method in [18], and the proposed method are summarized in Table 1.

Table 1 General workflow (A to D) of various time series analysis methods. Note that the response method used in this study does not include spherical harmonic modes from the radiation potential (OTS in this table stands for the original time series).

\begin{tabular}{cccc}
\hline Workflow & Harmonic Method & Response Method & This Study \\
\hline $\begin{array}{c}\text { Input data } \\
\text { (A) }\end{array}$ & Predetermined PTCs & $\begin{array}{c}\text { Computable TGP and } \\
\text { radiation potential data }\end{array}$ & $\begin{array}{c}\text { Computable } \\
\text { astronomical data }\end{array}$ \\
$\begin{array}{c}\text { Basis Function } \\
\text { (B) }\end{array}$ & $\begin{array}{c}\text { Sinusoids } \\
\text { of (A) }\end{array}$ & $\begin{array}{c}\text { Spherical harmonics } \\
\text { of (A) }\end{array}$ & $\begin{array}{c}\text { Eigenmodes } \\
\text { from EOFs of (A) }\end{array}$ \\
$\begin{array}{c}\text { Parameter Estimation } \\
\text { (C) }\end{array}$ & $\begin{array}{c}\text { Harmonic analysis } \\
\text { of (B) with OTS }\end{array}$ & $\begin{array}{c}\text { LLS } \\
\text { of (B) with OTS }\end{array}$ & $\begin{array}{c}\text { LLS } \\
\text { of (B) with OTS }\end{array}$ \\
$\begin{array}{c}\text { Prediction } \\
\text { (D) }\end{array}$ & $\begin{array}{c}\text { Linear combination } \\
\text { of (B) using (C) }\end{array}$ & $\begin{array}{c}\text { Linear combination } \\
\text { of (B) using (C) }\end{array}$ & $\begin{array}{c}\text { Linear combination } \\
\text { of (B) using (C) }\end{array}$ \\
\hline
\end{tabular}

\section{Performance evaluation}

In the case of the harmonic method, a computer program, written as part of the Institute of Ocean Sciences (IOS) Tidal Package to perform the VHTA, was used to analyze the training sea level data and reconstruct deep-ocean tide at the 57 DART stations [11]. A total number of 60 PTCs, with frequencies of up to about 8 cycles per day, consisting of 45 main constituents and 15 satellite constituents, were used to perform the VHTA (see http://www.dfo-mpo.gc.ca/science/data-donnees/tidal-marees/index-eng.html for more details). 
In the case of the response method, a computer program was written following the procedure described by Munk and Cartwright in 1966 [18]. However, astronomical ephemerides were replaced by the NASA JPL's DE405 solar system ephemeris for use with the proposed method in this study. Only spherical harmonic modes of the gravitational potential of $n=0$ to 2 and $m=0$ to $n$ (as found to be adequate in their original work in 1966) were used. Each mode with a maximum time lag of 6 days $(\Delta \tau=$ 1 day and $s=0$ to 6 , instead of $\Delta \tau=2$ days and $s=-3$ to +3 , as used in their work) was used to maintain the physical realizability. Moreover, to account for the fairness in performance comparison between the response method and the proposed method, in which the effect from solar radiation is not included, no radiation potential was included in the response method used in this study. This newly-written program, based on the response method, was also used to perform tidal reconstruction and prediction at the 57 DART stations.

In order to compare reconstruction performances between the proposed method, the response method, and the VHTA, RMS residuals between the training sea level data and the reconstructed oceanic tide data using these methods were computed. Deep-ocean tides at the 57 DART stations were also predicted for 1 year in advance and compared to their training sea level time series to compute RMS residuals of the predicted data. Reconstruction and prediction RMS residuals of using each method were also compared to examine their prediction performance and stability.

In this study, for easy comparison, reconstruction residuals will be reported as a percentage of the ratio between reconstruction RMS residuals and RMS deviation of the training climatic data, and prediction residuals will be reported as a percentage of the ratio between prediction RMS residuals and the training climatic data.

\section{Results and discussion}

\section{Astronomical variations}

In the case of DART 23219, the computed VTA and the training sea level eigenmodes were found to have distinct variations (Figures 4(a) to 4(I)). Time series orthogonalization of both VTA and sea level data also gave relatively smooth spectra compared to the spectrum of the original time series (Figures 5(a) to 5(I)). This smoothness clearly resulted from the property of correlation kernels that acted as bandpass filters, which were transparent to some specific variations. Since magnitudes of other variations were suppressed, to be nonsignificant, these correlation kernels usually produced eigenmodes having smoothed spectra.

Some VTA eigenmode pairs were found to have similar astronomical variations, as suggested by their spectra. This situation was also found in sea level eigenmodes. Notice that it is a property of the SSA, which is analogous to the harmonic method, in which summation of 2 orthogonal sinusoids of the same argument $x$ is equal to another sinusoid of the same frequency, but with different amplitude and phase, i.e., $A_{1} \cos (x)+A_{2} \sin (x)=\operatorname{sqrt}\left(A_{1}^{2}+A_{2}^{2}\right) \cos \left(x-\arctan \left(A_{2} / A_{1}\right)\right)$ [21]. Therefore, in this study, any pairs of eigenmodes with similar variations were expressed as the combined eigenmodes, but during the reconstruction and prediction, they were used separately as different predictors.

Five patterns of astronomical variations found in this study, which hereafter will be named according to 5 major PTCs $\left(M_{2}, S_{2}, N_{2}, K_{1}\right.$, and $\left.O_{1}\right)$, were identified for the first 10 VTA and sea level eigenmodes using the highest peaks found in their spectra (Figures 5(c) to 5(I) and Table 2). In the case of DART 23219, significant astronomical variations were of semidiurnal, diurnal, and long-term types, consistent with the results found by Munk and Cartwright, in which only spherical harmonic modes of up to semidiurnal variations ( $n=0$ to 2 ) could be adequately used [18]. Semidiurnal variations were found to have higher amplitudes than those of diurnal variations. Small sidebands contained in a cusp found around each dominant frequency also caused amplitude and frequency modulations. Note that, for example, the spectra of vertical GTA eigenmodes having a dominant variation of $M_{2}$, though similar, were not necessarily the same as those of sea level eigenmodes having the same dominant variation. This was because all VTA eigenmodes could contribute to the variation of each sea level eigenmode, but with different proportions, as indicated by multipliers $\left(m_{j, s}\right.$ in equation (17)) estimated using LLS. 
(a)

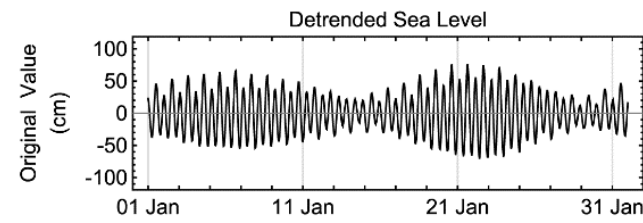

(c)

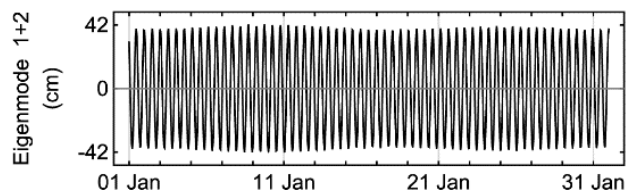

(e)

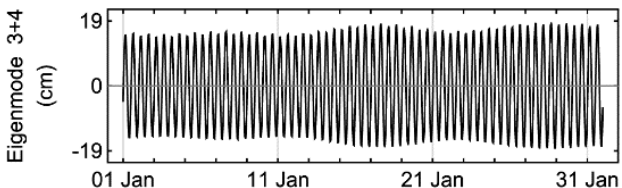

(g)

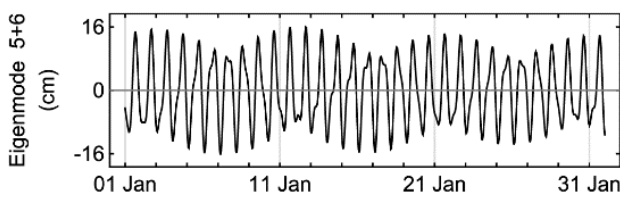

(i)

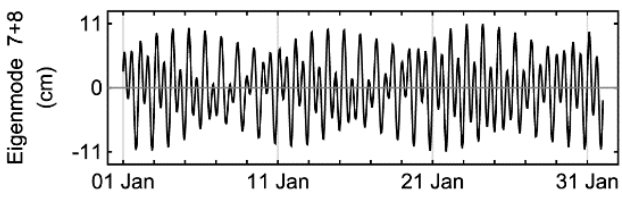

(k)

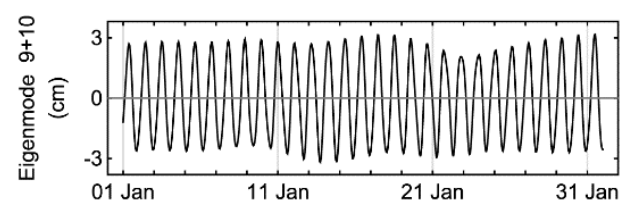

(b)

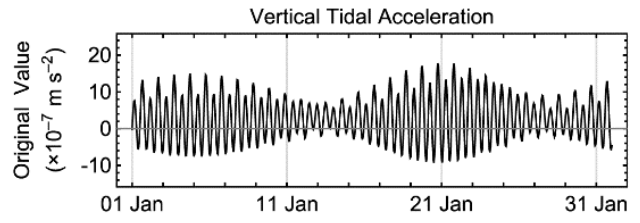

(d)

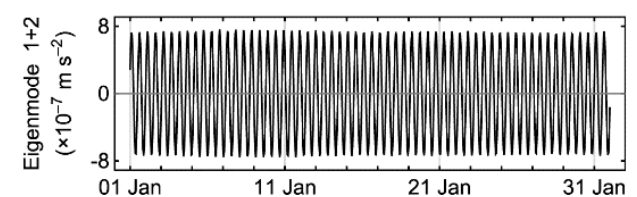

(f)

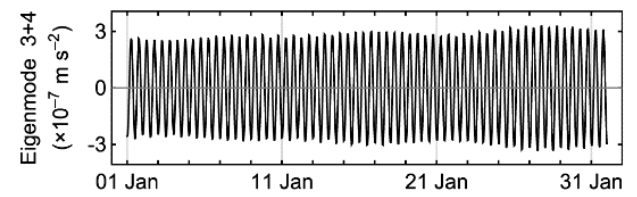

(h)



(j)
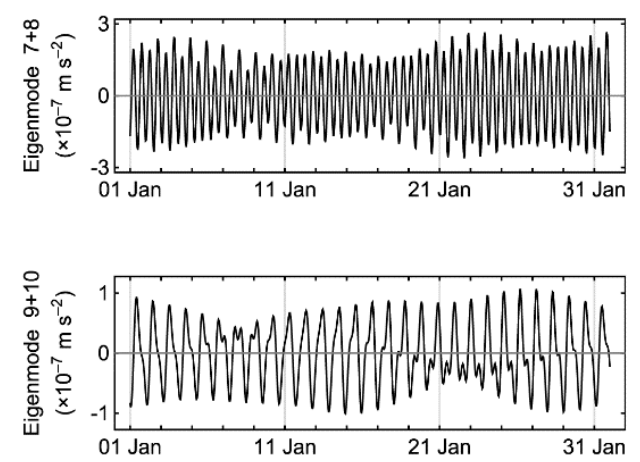

Figure 4 Time series plots of the training sea level data (a), the computed VTA data (b), and superposition of sea level eigenmodes (left side: (c), (e), (g), (i), and (k)) and VTA eigenmodes (right side: (d), (f), (h), (j), and (l)), which were identified to have the same astronomical variation at DART 23219 during January 2015. 
(a)

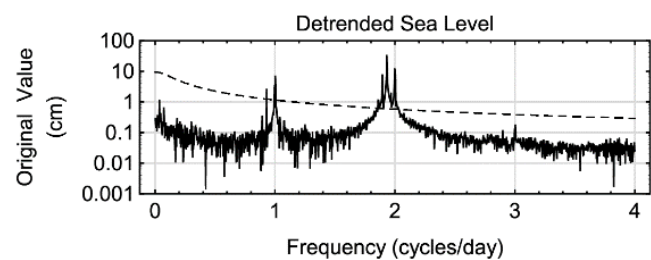

(c)

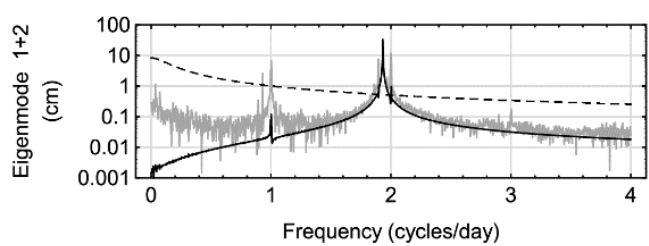

(e)

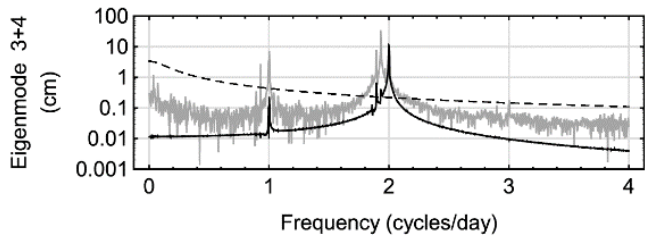

(g)

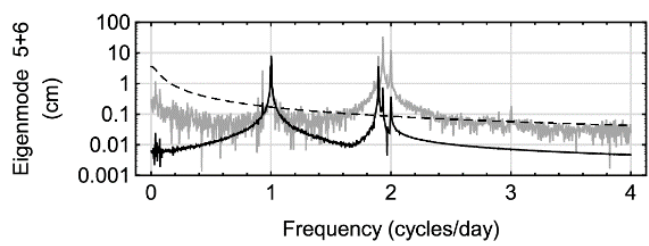

(i)

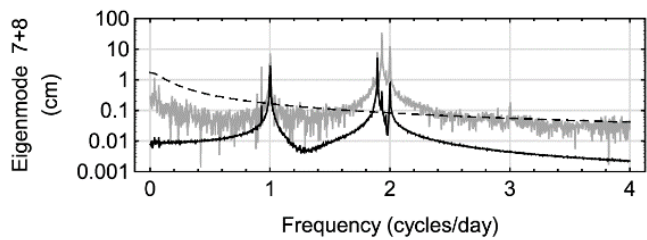

(k) $\frac{0}{5}$

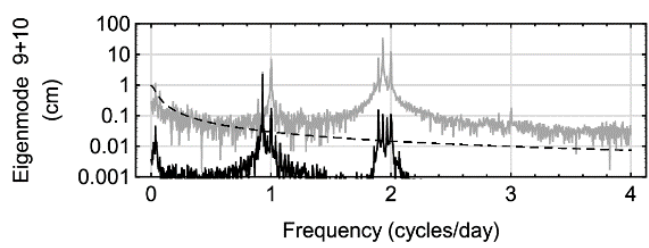

(b)

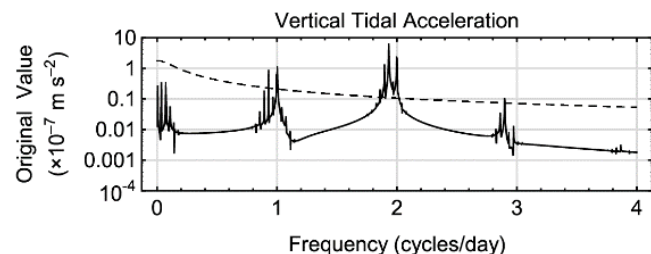

(d)

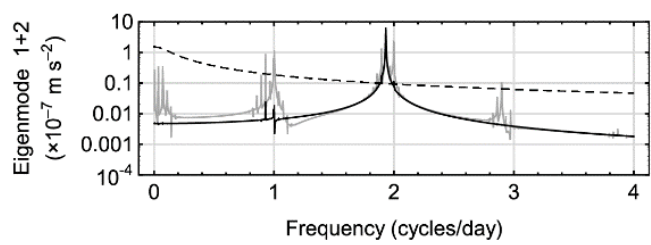

(f)

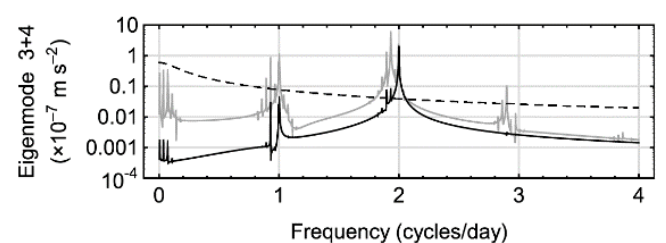

(h)

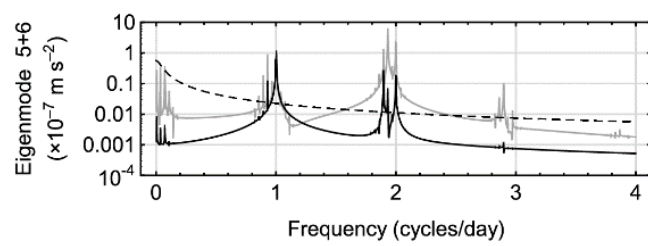

(j)

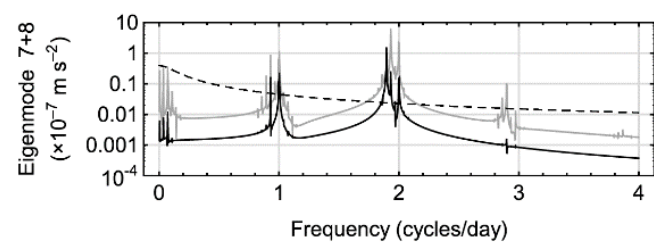

(I)

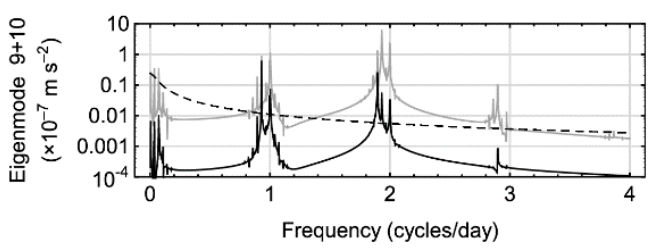

Figure 5 Harmonic analysis results using DFT (without performing satellite modulation) showing frequencies and amplitudes of the training sea level data (left side, black line in (a), and gray lines in (c), (e), (g), (i), and (k)), the computed VTA data (right side, black line in (b), and gray lines in (d), (f), (h), (j), and (1)), and superpositions of sea level and VTA eigenmodes which were identified to have the same astronomical variation at DART 23219. The dashed line in each graph represents the $95 \%$ confidence level of the red noise spectrum. 
http://wjst.wu.ac.th

Table 2 Dominant frequency, dominant period, and equivalent conventional PTCs of variations found in VTA eigenmode pairs. Each eigenmode pair is named according to its dominant PTC.

\begin{tabular}{cccrl}
\hline $\begin{array}{c}\text { Variation } \\
\text { Number }\end{array}$ & $\begin{array}{c}\text { Dominant Frequency } \\
\text { (cycles per day) }\end{array}$ & $\begin{array}{c}\text { Dominant Period } \\
\text { (hours) }\end{array}$ & $\begin{array}{c}\text { Equivalent Conventional } \\
\text { Principal Tidal Constituent }\end{array}$ \\
\hline 1 & 1.932231 & 12.4209 & $M_{2}$ & Principal Lunar Semidiurnal \\
2 & 2.000000 & 12.0000 & $S_{2}$ & Principal Solar Semidiurnal \\
3 & 1.895942 & 12.6586 & $N_{2}$ & Larger Lunar Elliptic Semidiurnal \\
4 & 1.002748 & 23.9342 & $K_{1}$ & Lunar Diurnal \\
5 & 0.929483 & 25.8208 & $O_{1}$ & Lunar Diurnal \\
\hline
\end{tabular}

It is noted that fluctuations of these VTA and sea level eigenmodes were similar to the superposition of harmonic constituents used to describe the oceanic tide (Figures 4(a) to 4(I)). However, by examining their frequencies and amplitudes using DFT (Figures 5(a) to 5(I) and Table 2), it can be shown that these eigenmodes were not the actual superposition of harmonic constituents (but closely resemble them), since each eigenmode always contained fluctuations from multiple harmonic constituents.

In the case of DART 23219, eigenmodes 1 and 2, for both VTA and sea level data contained a significant $M_{2}$ peak with its large cusp and a relatively small $S_{2}$ peak within the range of $M_{2}$ 's cusp (Figures 5(c) and 5(d)). Eigenmodes 3 and 4, for both VTA and sea level data, contained a significant $S_{2}$ peak, also with a large cusp, a relatively small $M_{2}$ peak, and an even smaller $N_{2}$ peak (Figures 5(e) and 5(f)). These first 2 astronomical variations clearly represented the average angular speed of the tidal influence from the Moon and the Sun projected onto the Earth's equator, while its cusp modulated both amplitude and frequency of this average angular speed and also resulted in the anharmonic behavior of this eigenmode pair. The presence of this cusp was analogous to satellite modulations used in the VHTA.

Eigenmodes 5 to 8, for both VTA and sea level data, were found to be nondependent with each other, due to the overlapping of their eigenvalues (Figure 3(c)). Eigenmodes 5 and 6 contained significant $K_{1} / S_{1}$ (these two PTCs were combined due to their similar frequencies, but hereafter will be called only $K_{1}$ ) and $N_{2}$ peaks with large amplitudes (a larger amplitude of $K_{1}$ than that of $N_{2}$ ) and their cusps (Figures 5(g) and 5(h)). Eigenmodes 7 and 8 also contained the same significant peaks with eigenmodes 5 and 6 , but with a larger amplitude of $N_{2}$ than that of $K_{1}$ (Figures 5(i) and 5(j)). The following 4 eigenmodes were a combination of astronomical variations that originated from the elliptical orbit of the Moon and the average angular speed of the Sun. Eigenmodes 9 and 10 were found to contain all significant PTCs mentioned before, with small amplitudes, and were dominated by $O_{1}$, which represented the average angular speed of the Moon on its mean orbital planes (Figures 5(k) and 5(I)).

Some long-period PTCs (such as lunar fortnightly $\left(M_{f}\right)$, lunisolar synodic fortnightly $\left(M_{s f}\right)$, lunar monthly $\left(M_{m}\right)$, solar semiannual $\left(S_{s a}\right)$, and solar annual $\left(S_{a}\right)$ ) which were also found in both VTA and sea level eigenmodes had small and obvious amplitudes but were not significant compared to the $95 \%$ confidence levels of their red noise spectra (Figures 5(c) to 5(I)). The red noise spectra were estimated from temporal functions generated using the first-order autoregressive process, or AR(1), in which random process parameters were fitted with each eigenmode. The $95 \%$ confidence level of the red noise spectra associated with each eigenmode was estimated by simulating 1,000 paths of the fitted AR(1) process, then computing the $95^{\text {th }}$ percentile value of amplitudes at each frequency.

These suggest that the harmonic method usually fits most of the small amplitudes PTCs to variations contained in the red noise. This situation also indicates that, although the estimated PTCs can reduce RMSEs of the predicted time series in practice, they have no physical meaning, since they were fitted to the red noise. However, in the case of eigenmodes obtained from the proposed method (including spherical harmonic modes obtained from the response method), although some peaks which obviously appeared in their spectra were not significant, they were part of the predictors' time series, representing the same pattern of astronomical variation which could not be separated (unlike PTCs used in the harmonic method, which are always separable) [21]. 


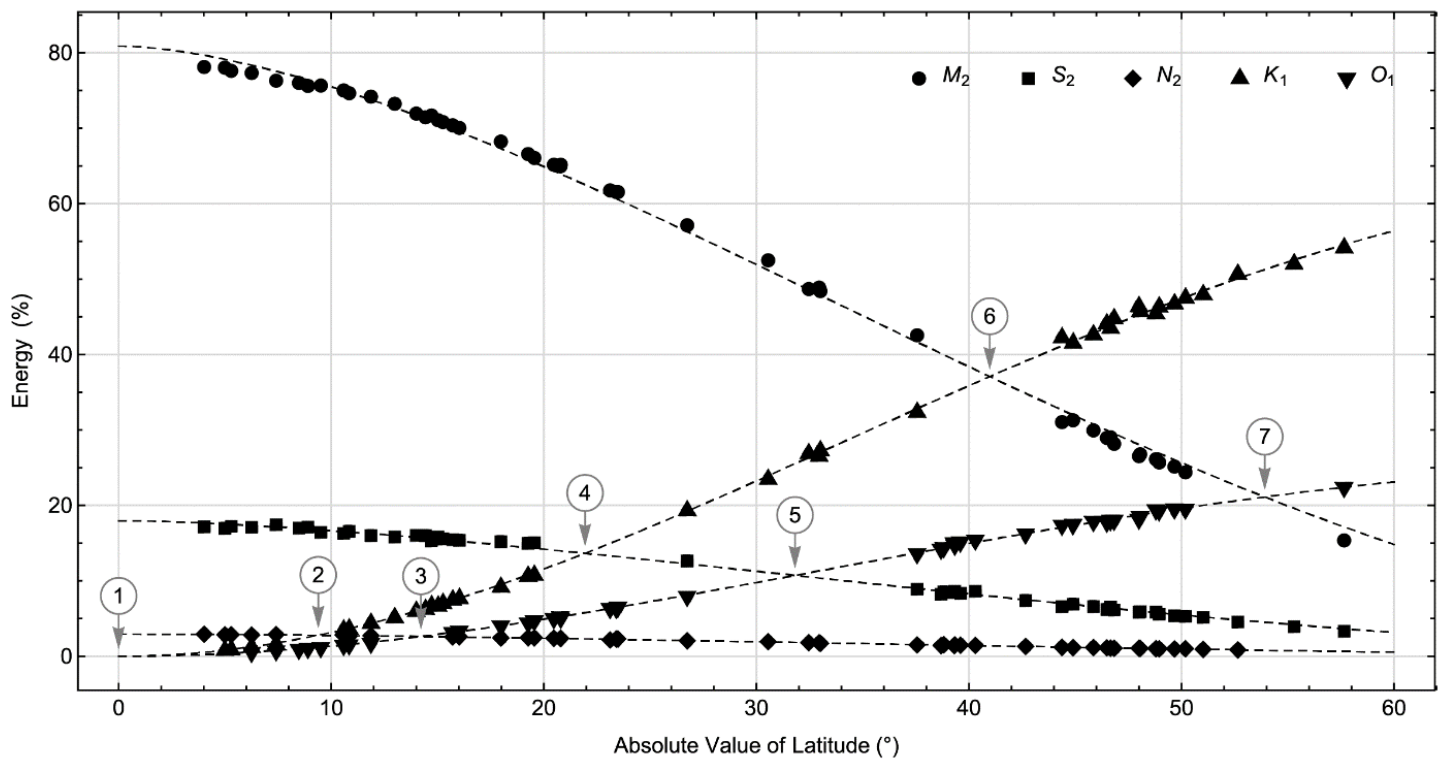

Figure 6 A scatter plot showing energies of astronomical variations found in VTA eigenmodes (markers having different shapes). Dashed lines represent the estimated energies of these variations at any latitude fitted using the squared cosine function. Their total energies (summation of all dashed lines) are usually concentrated at around $99 \%$, but not less than $96 \%$ (not shown in this graph). Regions in which any 2 dashed lines cross together are zones in which mixed characteristics appeared in some eigenmodes due to their overlapping eigenvalues.

A scatter plot of astronomical variations' energies shows zonal patterns similar to those of spherical harmonic modes used in the response method, in which amplitudes of semidiurnal variations are generally highest at the equator and vanish at both poles (and vice versa for diurnal variations) (Figure 6). The total energies of the first 10 VTA eigenmodes at the 57 DART stations were usually concentrated at around $99 \%$, but not less than $96 \%$. However, there were some latitudes in which mixed characteristics appeared in some eigenmodes (Figure 6). VTA eigenmodes at some DART stations which were located in these zones usually experienced overlapping of eigenvalues.

Energies of the first 10 sea level eigenmodes were found to differ between stations (Figures 7(a) to 7(e)). However, their total energies were usually to be more than about $97 \%$. Energies of the same astronomical variation in the VTA and the sea level data at the same station were found to be different. These energy differences, which were possibly caused by the effect from the ocean bathymetry, suggest that energies computed using time series orthogonalization based on the SSA approach can be used to quantitatively estimate these responses.

In the case of DART 23219, the first 10 VTA eigenmodes, with a total energy of $98.97 \%$, were used to predict the superposition time series of the first 10 sea level eigenmodes, with a total energy of 99.31\% (Figure 8). The energy of each astronomical variation was found to change slightly, but its order in both the VTA and the sea level data was the same. However, changes in astronomical variation order in other stations were also found and were caused by the change in the oceanic tide's response to the VTA. 
(a)

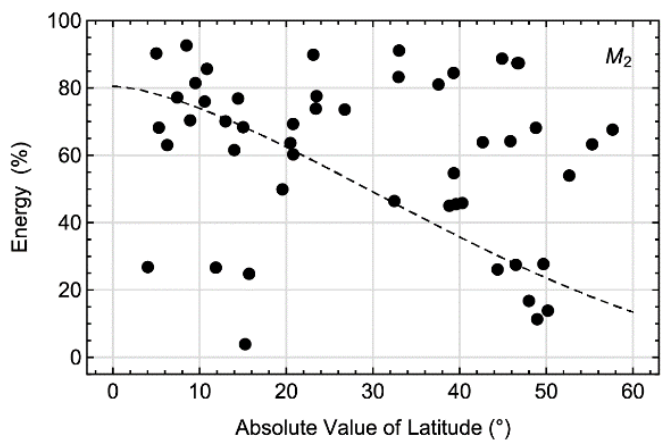

(c)



(e)

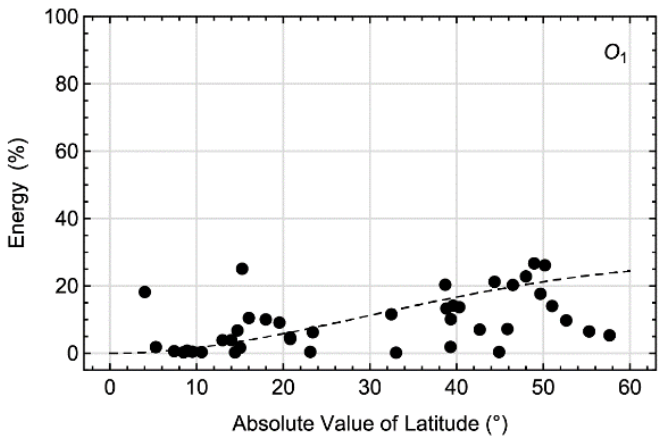

(b)

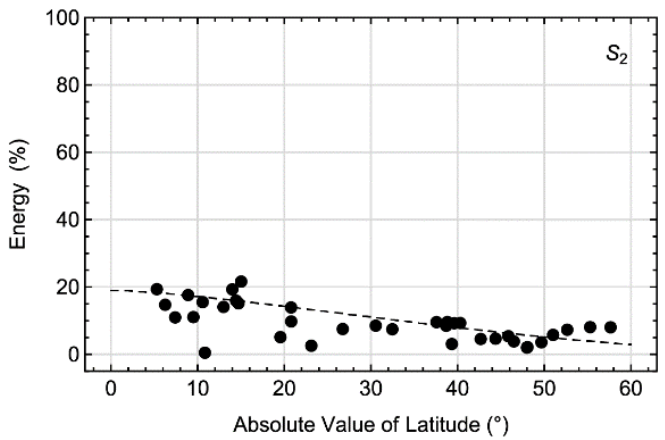

(d)

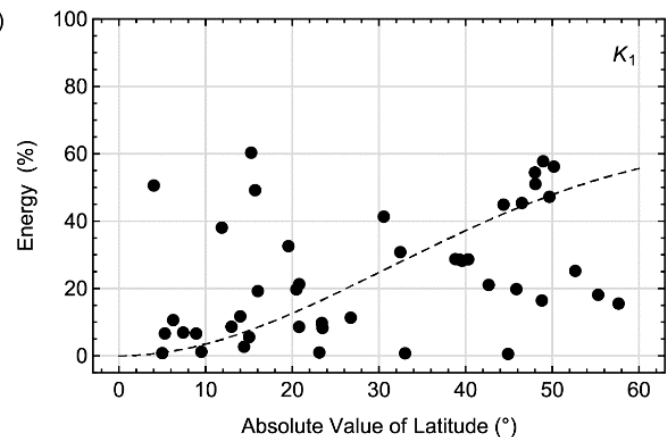

Figure 7 Scatter plots showing energies of 5 astronomical variations found in sea level eigenmodes at 57 DART stations (dots). These values deviate from their latitude-dependent trends found in VTA eigenmodes (dashed lines, as also shown in Figure 6), which were empirically-fitted using energies of astronomical variations found in VTA eigenmodes.

Since DART 23219 is located in the middle of a relatively deep sea (with a seafloor depth of about $3.3 \mathrm{~km}$ ), the change in energies of each sea level eigenmodes might be originated from the northern tip of the Ninety-East Ridge and the Andaman and Nicobar Islands, which block seawater circulation and also dissipate tidal energies of each astronomical variations at different proportions. This resulted in slightly increasing or decreasing sea level eigenmode energies (Figure 8). 
http://wjst.wu.ac.th

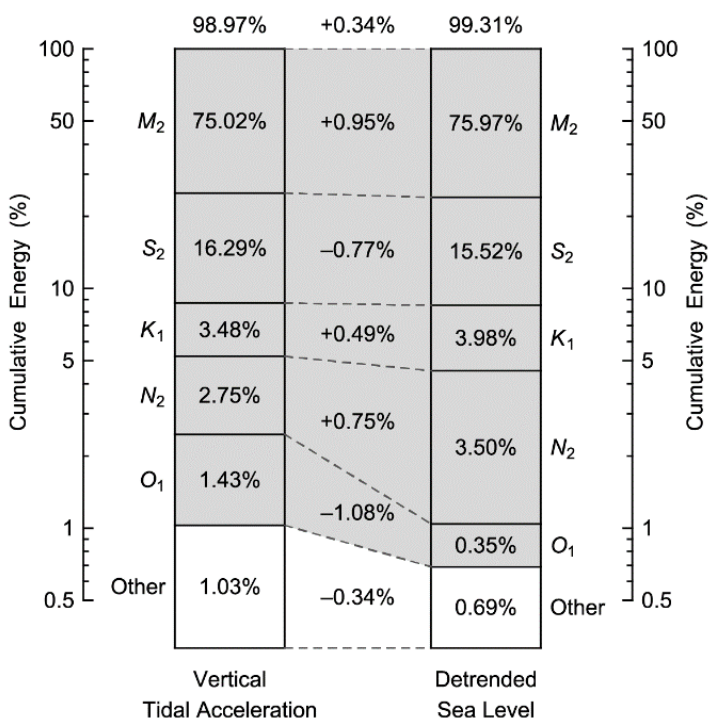

Figure 8 Diagram showing individual energy (in logarithmic scale) and energy changes from VTA to sea level eigenmodes at DART 23219.

\section{Reconstruction and prediction performance}

Tidal reconstruction results using the VHTA, the response method, and the proposed method at the 57 DART stations indicate that all of the mentioned methods gave slightly different reconstruction accuracies. As compared to the VHTA, the response method was found to give higher reconstruction RMS residuals (Figure 9(a)), while the proposed method gave similar reconstruction RMS residuals (Figure 9(c)). This implies that, when comparing the response method and the proposed method, the latter also gave better reconstruction accuracy (Figure 9(e)). In the case of prediction performances, prediction RMS residuals of the response method were still found to be lower than those of the VHTA and the proposed method (Figures 9(b) and 9(f)). However, prediction RMS residuals using the proposed method were found to be slightly lower than those of the VHTA, which indicates that the proposed method can give slightly better prediction accuracy than the VHTA (Figure 9(d)).

Prediction RMS residuals of the 57 DART stations using the VHTA, the response method, and the proposed method were also found to be similar to their reconstruction RMS residuals (Figures 10(a) to 9(c)). This implies that all methods used in this study can give unpropagated prediction RMS residuals or stable prediction accuracy (or, if this residual propagation really exists, it cannot be seen using 1 year prediction data). However, as can be implied from Figure 9(d), most of the VHTA prediction residuals appear to be slightly higher than those of the proposed method. This indicates that the VHTA, in fact, gives prediction results that are slightly deviated from the actual oceanic tide over time. Although this deviation cannot be obviously seen in Figure 10(a), it can be seen when compared to the use of another method, as in Figure 9(d).

In addition to the stability of each prediction method, the propagation of prediction RMS residuals can also be caused by a change of the environmental conditions, such as local bathymetry, ocean circulation, or wind systems which alter the sea level response to the VTA. For example, the change of location of DART 23219 after instrument redeployment caused the prediction residuals after June 2016 to obviously increase (Figure 2(a)). This increase of prediction RMS residuals from reconstruction RMS residuals can be found using all methods used in this study (Figures 10(a) to 10(c)). The oceanic tide response, which was estimated from the training data using these methods, cannot be used to accurately predict deep-ocean tide after station redeployment at a different location. 
(a)

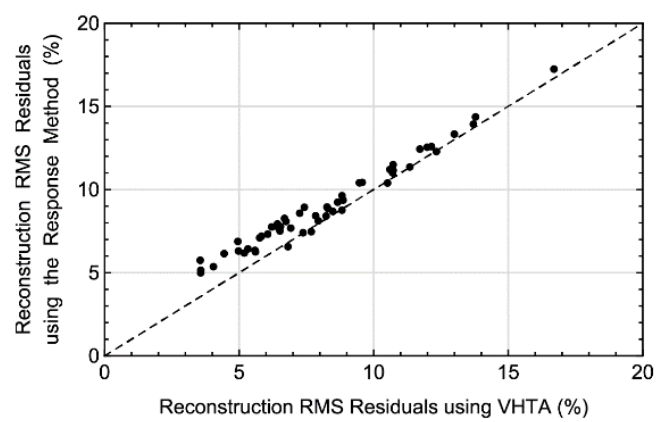

(c)

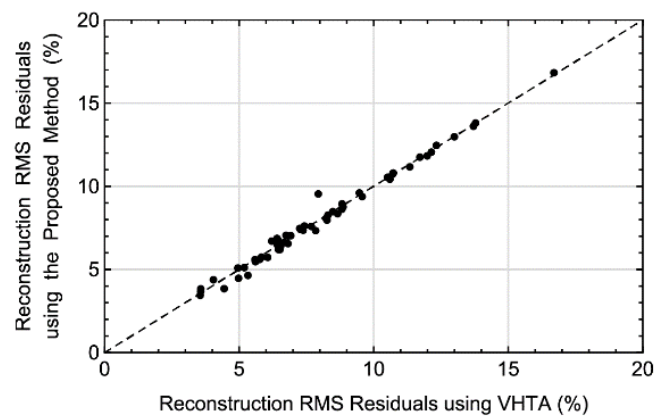

(e)

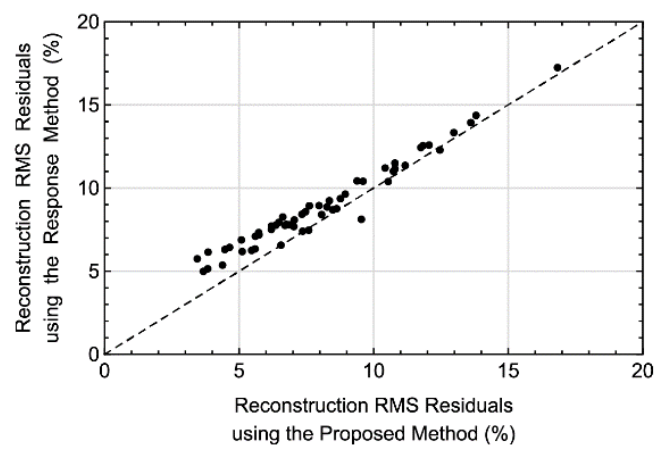

(b)

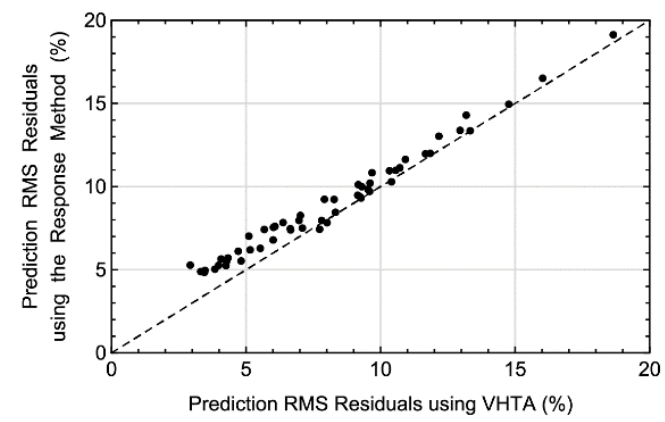

(d)

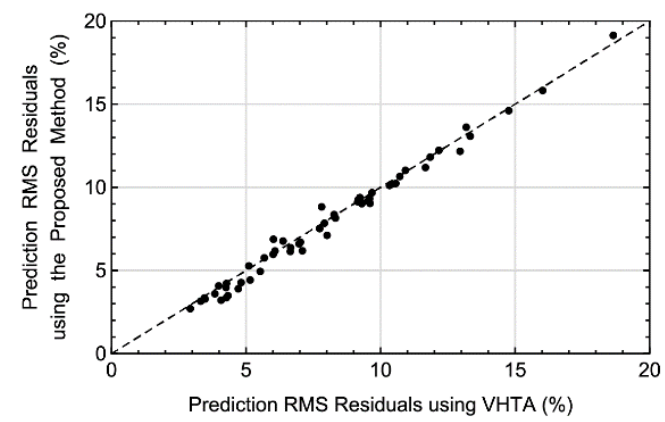

(f)

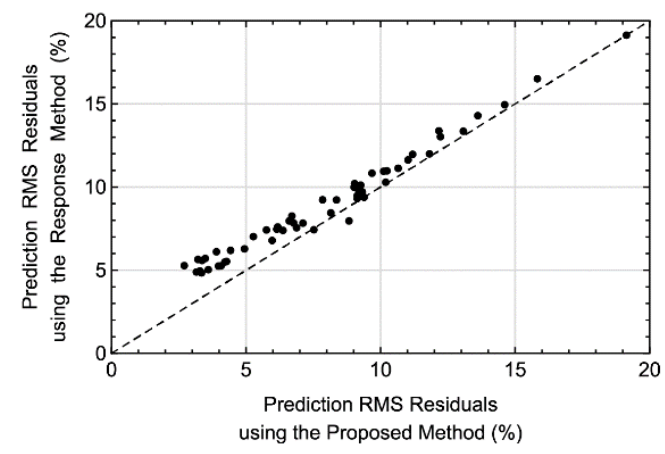

Figure 9 Scatter plot showing reconstruction (left side) and prediction (right side) RMS residuals at 57 DART stations between the VHTA and the response method ((a) and (b)), between the VHTA and the proposed method ((c) and (d)), and between the proposed method and the proposed method ((e) and (f)). Dashed lines represent a one-to-one function in which RMS residuals between 2 methods are equal. Reconstruction and prediction RMS residuals of using the response method were found to be higher than the other 2 methods (dots in (a), (b), (e), and (f) are above the dashed lines, especially when RMS residuals of using the VHTA or the proposed method are lower than $10 \%$ ). While, for the VHTA and the proposed method, their reconstruction RMS residuals are similar (black dots in (c) are at around the dashed line), the prediction RMS residuals of the proposed method are slightly lower than those of the VHTA (black dots in (d) are just below the dashed line). 
(a)

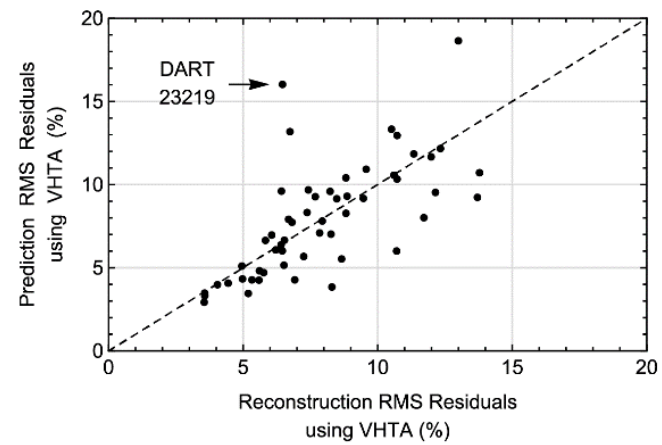

(c)

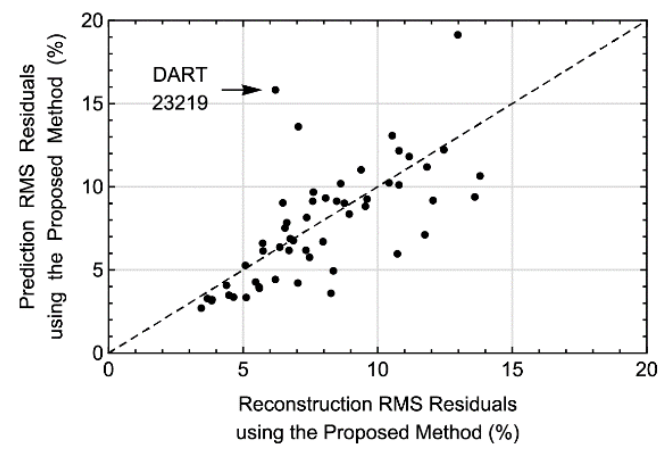

(b)

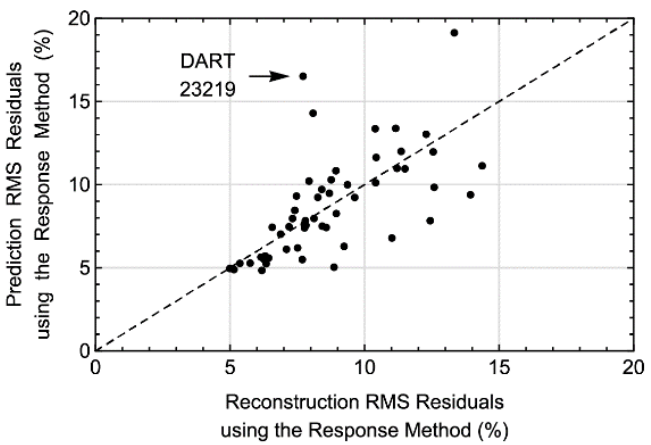

Figure 10 Scatter plot showing reconstruction and prediction RMS residuals of 57 DART stations using the VHTA (a), the response method (b), and the proposed method (c). Dashed lines represent a one-to-one function in which reconstruction and prediction RMS residuals are equal. The change in oceanic tide response to the VTA can be detected as the change of prediction RMS residuals from reconstruction RMS residuals. For example, the change of BPR deployment location at DART 23219 changed its oceanic tide response, which can be found as an abrupt increase of the prediction RMS residuals from the reconstruction RMS residuals (from 6.46 to $16.02 \%$ using the VHTA, 7.71 to $16.51 \%$ using the response method, and 6.20 to $15.83 \%$ using the proposed method).

\section{Conclusions}

Time series orthogonalization based on the SSA approach proposed in this study can be used to decompose any time series into orthogonalized components or eigenmodes. These eigenmodes represent the projection of data onto the scaled principal axes, which can be used as a set of temporal state variables of each time series. This approach has the advantage that the combination of all eigenmodes adds up to the original time series, and the combination of the first few eigenmodes approximates a time series.

Time series orthogonalization can also be applied to quantitatively estimate the change of energy (or the explained variation) between VTA and sea level eigenmodes of the same astronomical variation. This approach has an advantage over the response method in that it uses PCA to decompose both VTA and sea level time series and obtain their eigenvalues or energies of eigenmodes, while the response method only gives eigenvalues of gravitational potential from spherical harmonics expansion. This causes the response method to be unable to be used in estimating the energy change of each astronomical variation.

The oceanic tide prediction proposed in this study can be used to estimate the response of deepocean tide to the VTA using short-term sea level data with the unpropagated prediction residuals. Moreover, prediction performances of using 10 VTA eigenmodes to predict the actual deep-ocean tide time series were found to be better than those of the VHTA and the response method. This suggests that the proposed method can probably be used in the early warning of tsunamis because sea level data 
obtained from buoys deployed in the open ocean often contain missing values. Using traditional harmonic analysis to predict these short-term data can probably give erroneous results and lower the performance of tsunami detection.

The proposed method can also be used to correctly predict the oceanic tide for historical purposes, as well as to study the paleoclimate $[9,10]$. This method does not rely on the performance of the required astronomical arguments of the Moon and the Sun (as in traditional harmonic analysis) but directly uses computed positions which can also include those of other planets. Therefore, if the algorithm used to compute the position of celestial objects is accurate enough, then the predicted oceanic tide results using the proposed method are also guaranteed to be accurate. Also, if this method has given accurate oceanic tide in the past, it can also give accurate prediction results in the future, which can be used to predict other phenomena such as small-magnitude earthquakes in some areas [1,2]. The SSA approach can also be applied to study the effect of sea level rise of non-astronomical origins $[7,8]$. This can be done by removing the fluctuation of the oceanic tide computed using the proposed method from a sea level time series, in which the tide-removed time series can be estimated for the trend, and the energy obtained during the computation indicates its variation.

This study only assumes that the VTA contributes to the fluctuation of the deep-ocean tide. In the case of the shallow-water tide, increasing the fragments' timespan to cover long-term variations and the use of more components, including zonal and meridional tidal acceleration, is recommended to fully estimate the movement of water parcels and account for the effect of local bathymetry.

This method can also be used with other types of time series (not limited only to VTA and sea level data), since it was utilized with the flexibility to do so. The general purpose of using the proposed method is to quantitatively assess linear and also weak nonlinear responses of an output to an input time series or quantitatively assess the relationship between 2 associated time series. This reconstruction and prediction method is practical and flexible to be applied in analyzing the properties and dynamics of any time series.

\section{References}

[1] Y Tan, M Tolstoy, F Waldhauser and D Bohnenstiehl. Tidal triggering of microearthquakes over an eruption cycle at 950’N East Pacific Rise. Geophys. Res. Lett. 2018; 45, 1825-31.

[2] C Scholz, Y Tan and F Albino. The mechanism of tidal triggering of earthquakes at mid-ocean ridges. Nat. Commun. 2019; 10, 2526.

[3] L Padman, M Siegfried and H Fricker. Ocean tide influences on the Antarctic and Greenland ice sheets. Rev. Geophys. 2018; 56, 142-84.

[4] A Robel, V Tsai, B Minchew and M Simons. Tidal modulation of ice shelf buttressing stresses. Ann. Glaciol. 2017; 58, 12-20.

[5] J Shi, X Yin, Q Shu, B Xiao and F Qiao. Evaluation on data assimilation of a global high resolution wave-tide-circulation coupled model using the tropical Pacific TAO buoy observations. Acta Oceanol. Sin. 2018; 37, 8-20.

[6] A Carrasco, T Plomaritis, J Reyns, Ó Ferreira and D Roelvink. Tide circulation patterns in a coastal lagoon under sea-level rise. Ocean Dyn. 2018; 68, 1121-39.

[7] L Wright, J Syvitski and C Nichols. Sea level rise: Recent trends and future projections. In: L Wright and C Nichols (Eds.). Tomorrow's Coasts: Complex and Impermanent. Springer, Cham, 2019, p. 47-57.

[8] R Nerem, B Beckley, J Fasullo, B Hamlington, D Masters and G Mitchum. Climate-change-driven accelerated sea-level rise detected in the altimeter era. Proc. Natl. Acad. Sci. 2018; 115, 2022-5.

[9] F Motoyama, M Tsunakawa and H Takahashi. Tidal resonance of higher-degree modes in the early Earth's ocean and its effect on the lunar-orbit evolution. In: Proceedings of the $20^{\text {th }}$ EGU General Assembly Conference, Vienna, Austria, 2018, p. 18315.

[10] $\mathrm{T}$ Weber and $\mathrm{M}$ Thomas. Tidal dynamics and their influence on the climate system from the Cretaceous to present day. Glob. Planet. Change 2017; 158, 173-83.

[11] M Foreman, J Cherniawsky and V Ballantyne. Versatile harmonic tidal analysis: Improvements and applications. J. Atmos. Ocean. Technol. 2009; 26, 806-17. 
http://wjst.wu.ac.th

[12] W Munk and B Bills. Tides and the climate: Some speculations. J. Phys. Oceanogr. 2007; 37, 13547.

[13] G Godin. The Analysis of Tides. University of Toronto, 1972.

[14] R Mousavian and M Hossainali. Detection of main tidal frequencies using least squares harmonic estimation method. J. Geod. Sci. 2012; 2, 224-33.

[15] M Yousefi and M Hossainali. Analyzing the tidal frequency content using Karhunen-Loève Expansion technique. J. Geod. Sci. 2013; 3, 79-86.

[16] D Cartwright. A unified analysis of tides and surges round north and east Britain. Philos. Trans. R. Soc. A Math. Phys. Eng. Sci. 1968; 263, 1-55.

[17] D Cartwright. Tides: A Scientific History. Cambridge University Press, 1999.

[18] W Munk and D Cartwright. Tidal spectroscopy and prediction. Philos. Trans. R. Soc. London. Ser. A, Math. Phys. Sci. 1966; 259, 533-81.

[19] G Groves and R Reynolds. An orthogonalize convolution method of tide prediction. J. Geophys. Res. 1975; 80, 4131-8.

[20] J Beckers and M Rixen. EOF calculations and data filling from incomplete oceanographic datasets. J. Atmos. Ocean. Technol. 2003; 20, 1839-56.

[21] N Golyandina and A Zhigljavsky. Singular Spectrum Analysis for Time Series. $1^{\text {st }}$ ed. Springer, 2013.

[22] D Cartwright and R Tayler. New computations of the tide-generating potential. Geophys. J. Int. 1971; 23, 45-73.

[23] G Kaplan, J Hughes, P Seidelmann, C Smith and B Yallop. Mean and apparent place computations in the new IAU system. III - Apparent, topocentric, and astrometric places of planets and stars. Astron. J. 1989; 97, 1197-210.

[24] M Ghil, MR Allen, MD Dettinger, K Ide, D Kondrashov, ME Mann, AW Robertson, A Saunders, Y Tian, F Varadi and P Yiou. Advanced spectral methods for climatic time series. Rev. Geophys. 2002; 40, 1003-43.

[25] R Vautard and M Ghil. Singular spectrum analysis in nonlinear dynamics, with applications to paleoclimatic time series. Phys. D Nonlinear Phenom. 1989; 35, 395-424. 